\title{
Tectonic arrangement of $\mathrm{BaCO}_{3}$ nanocrystals into helices induced by a racemic block copolymer
}

\author{
SHU-HONG YU1*, HELMUT CÖLFEN²* KLAUS TAUER ${ }^{2}$ AND MARKUS ANTONIETTI2* \\ 'Division of Nanomaterials and Chemistry, Hefei National Laboratory for Physical Sciences at Microscale, Department of Chemistry, and Department of Materials Science and \\ Engineering, University of Science and Technology of China, Hefei 230026, Anhui, PR China \\ 2Department of Colloid Chemistry, Max Planck Institute of Colloids and Interfaces, MPI Research Campus Golm, D-14424, Potsdam, Germany \\ *e-mail: shyu@ustc.edu.cn; coelfen@mpikg-golm.mpg.de; pape@mpikg-golm.mpg.de
}

$\mathbf{M}$ orphosynthesis strategies inspired by biomineralization processes gives access to a wide range of fascinating and useful crystalline mesostructures ${ }^{1-3}$. Biomimetic synthesis of inorganic materials with complex shapes can now be used to control the nucleation, tensorial growth, and alignment of inorganic crystals in a way previously not practicable ${ }^{3}$. Double hydrophilic block copolymers (DHBCs) ${ }^{4}$ consisting of a hydrophilic block strongly interacting with inorganicminerals, and a non-interacting hydrophilic block, were recently introduced for the control of mineralization reactions. DHBCs are 'improved versions' of the previously used polyelectrolytes or amphiphiles and are extraordinarily effective in crystallization control $^{5-9}$. Here, we report on the formation of helices of achiral $\mathrm{BaCO}_{3}$ nanocrystals in the presence of a racemic DHBC suggesting that a helical alignment can be induced by racemic polymers through selective adsorption on the (110) face of nanocrystals. This mechanism is the key for a better understanding of the self-assembly of chiral organic-inorganic superstructures that don't follow a direct template route.

Chiral mineralized structures as found in marine and snail shells have attracted intense interest for a long time. Besides nanofibre ${ }^{10}$, tubes and ribbons, and novel helical inorganic nanostructures ${ }^{11,12}$ have been highlighted due to the expectation of their unusual applications. Some work has indicated that such fibres might catalyse chiral addition reactions in a highly enantiospecific way ${ }^{13}$. Current synthesis approaches for these unusual helical structures mainly rely on the direct templating effect from some specific supramolecular nanoribbons ${ }^{11}$, screw dislocations ${ }^{12}$, transcription from chiral gelators ${ }^{14}$ or in an unknown way from chiral acidic polypeptides ${ }^{15}$. A helical superstructure of $\mathrm{CaCO}_{3}$ particles with micrometre size was occasionally observed by Gower et al..$^{16}$ using chiral as well as achiral polyaspartate additives, however, the origin of these polycrystalline structures is not yet well understood. In addition, helical micrometre-sized filaments of silica-barium carbonates were synthesized in alkaline sodium silicate solutions $s^{17,18}$. Helical morphologies of several triclinic crystals were created from a gel matrix based on twisting with a constant angle of twinned crystals ${ }^{19}$

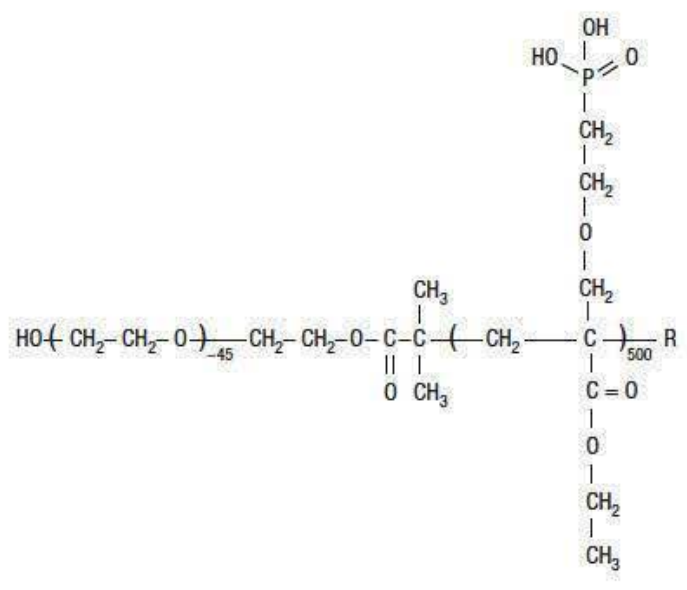

Figure 1 Chemical structure of PEG- $b$-DHPOBAEE. $R$ is either another PEG block or a hydrogen atom depending on the termination mode during polymerization.

and micrometre-sized $\mathrm{SrCO}_{3}$ helical forms were observed when the crystals grew in silica gel ${ }^{20}$.

The first helical structures, formed by molecular tectonics consisted of elongated $\mathrm{CaCO}_{3}$ crystals formed in the presence of chiral aspartic acid and phosphoserine copolymers ${ }^{15}$. The question remains of how a chiral polymer with contour lengths in the nanometre range influences the tectonic arrangement of mineral particles on the micrometre scale. Thus, it is of great interest to explore the mechanisms that lead to such delicate structures.

A newly designed DHBC, polyethyleneglycol- $b$ - [(2-[4-dihydroxy phosphoryl]-2-oxabutyl) acrylate ethyl ester] (PEG- $b$-DHPOBAEE), synthesized by using a poly(ethylene glycol)-azo-initiator ${ }^{21}$, was applied during the crystallization process of $\mathrm{BaCO}_{3}$ (Fig. 1, also see Supplementary information). Owing to the random nature of 


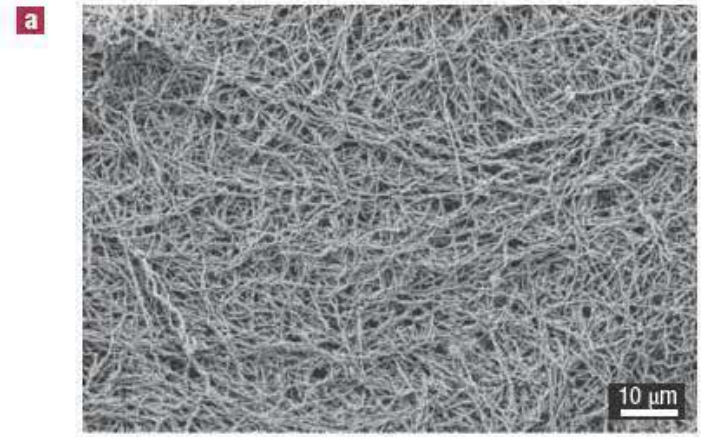

Б

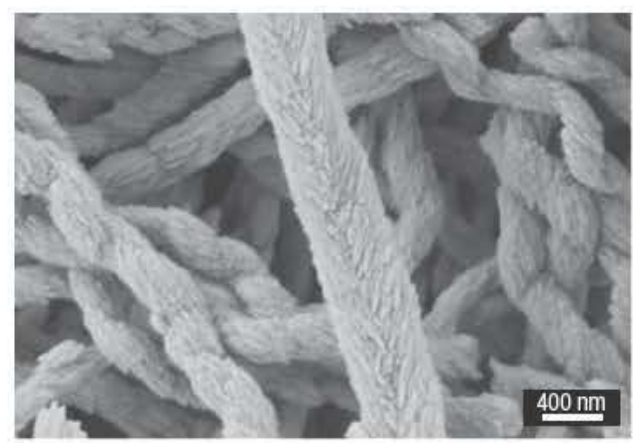

Figure 2 Helical nanoparticle superstructures. a, The helical fibres formed at room temperature with PEG-b-DHPOBAEE, $1 \mathrm{~g} \mathrm{l}^{-1}$, starting $\mathrm{pH} 4,\left[\mathrm{BaCl}_{2}\right]=10 \mathrm{mM}$. b, Detailed surface structure.

the applied radical polymerization in the absence of coordination agents, the polymer is not expected to show specific tacticity. Poly(methyl methacrylate) was reported to be highly syndiotactic ( $75 \%$ syndiotactic, $25 \%$ atactic) due to substituent repulsion in the monomer unit ${ }^{22}$ and this should especially be true for our polymer with its bulky substituents. Our polymer was optically inactive with or without added $\mathrm{Ba}^{2+}$ ions, which does not $a$ priori exclude the formation of helices in solution ${ }^{23,24}$, but makes it very unlikely as the synthesized DHBC shows no tendency to form superstructures by aggregation as shown by analytical ultracentrifugation. Also, molecular modelling suggests a straight, but non-helical structure with the phosphonate groups predominately located on one side of the molecule (Supplementary Information, Fig. S1). Although the modelling was done in vacuum, it can be assumed that hydration will only increase the steric constraints justifying the model of a stiff polymer.

$\mathrm{BaCO}_{3}$ precipitation was carried out through decomposition of ammonium carbonate as described for $\mathrm{CaCO}_{3}$ (ref. 25) with $1 \mathrm{~g} \mathrm{l}^{-1}$ polymer additive in the $\mathrm{BaCl}_{2}$ solution. Extremely long $\mathrm{BaCO}_{3}$ fibres with a diameter of $200-500 \mathrm{~nm}$ and lengths as long as some millimetres are obtained after mineralization for two weeks (Fig. 2a). Astonishingly, most fibres (more than $90 \%$ from 200 counted particles) possess helicity, with a very tiny fraction of straight herringbone structures (less than 10\%). The number ratio of right- and left-handed helices is 50:50, as obtained from counting 200 helices. The obvious helical structure of the fibres is composed of very fine, homogeneous brick-like elongated nanocrystals about $200 \mathrm{~nm}$ in length and $30 \mathrm{~nm}$ in diameter, which self-assemble towards helices (Fig. 2b). X-ray diffraction analysis (Supplementary Information, Fig. S3) confirmed pure witherite $\left(\mathrm{BaCO}_{3}\right)$ with cell constants $a=6.43, b=5.32, c=8.90 \AA$ (orthorhombic structure; space group Pnma). Default experiments in the absence of block
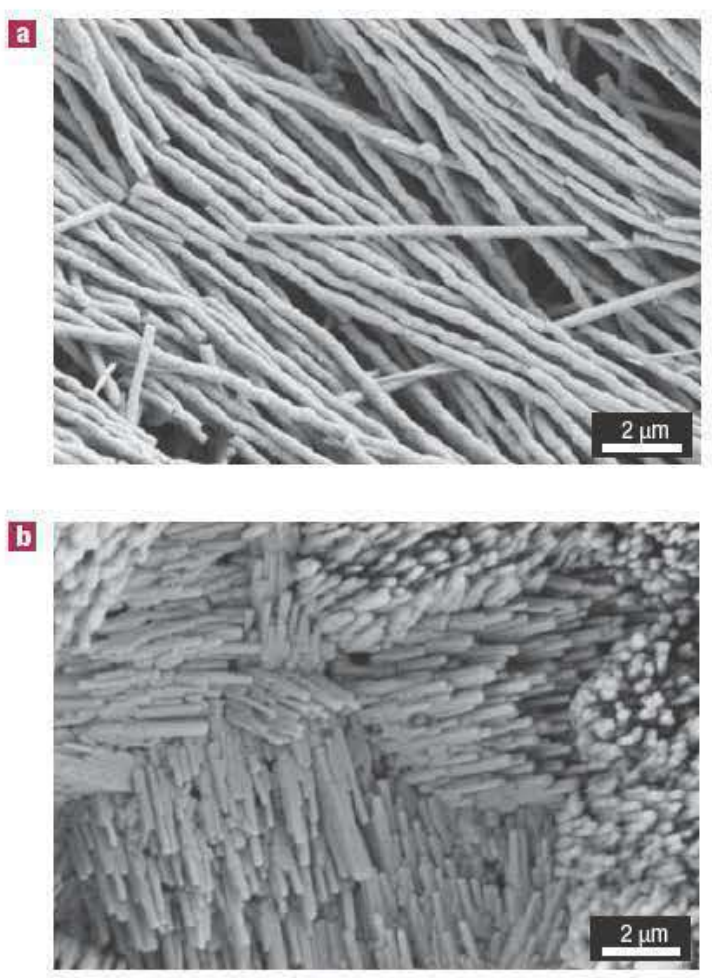

Figure 3 Change of helical pitch. a, $\mathrm{BaCO}_{3}$, [polymer] $=1 \mathrm{gl}^{-1}$, starting $\mathrm{pH} 5.6$, $\left[\mathrm{BaCl}_{2}\right] 10 \mathrm{mM} . \mathrm{b}, \mathrm{BaCO}_{3}$ arrays, [polymer] $=2 \mathrm{gl}^{-1}$, starting $\mathrm{pH} 4,\left[\mathrm{BaCl}_{2}\right]=10 \mathrm{mM}$. Overview in Supplementary Information, Fig. S4d.

copolymers under similar conditions only produce dendritic witherite crystals (Supplementary Information, Fig. S4a).

The formation of a helical superstructure is surprising because the polymer is obviously not helical or optically active, which previously accounted for tectonic helix formation ${ }^{15}$. In addition, it has to be pointed out that the structural dimensions of the observed inorganic helices are a magnitude bigger than the molecular dimension of an eventually formed block copolymer helix so that a direct polymer template effect can be excluded. Analysis of the early stages of the reaction after five hours revealed only nearly spherical 20-50-nm amorphous nanoclusters (Supplementary Information, Fig. S2), implying that the fibres were developed from such polymerstabilized nanoclusters through a mesoscale transformation process ${ }^{2,26}$ or a directed aggregation process under polymer control.

A closer observation of Fig. $2 \mathrm{~b}$ reveals that the helicity is created by the mutual orientation of the single nanocrystals with an axial ratio about seven, that is, a bend-staggered arrangement of the nanocrystals is the predominant mutual packing motif, where elongated primary crystals are arranged in a parallel manner along their elongated crystal faces. This also corresponds to the general finding that rods attract each other along their elongated sides forming bundle structures ${ }^{2728}$. Thus, the aligned primary crystals appear to share the same crystallographic axis (Supplementary Information, Fig. S4b,c). It is noteworthy to stress that most of the elongated primary crystals have to be aligned in an almost parallel fashion, as otherwise a helix structure would be significantly disturbed, although the alignment is not perfect (Supplementary Information, Fig. S4b,c).

A herringbone-like supracrystalline packing motif (Fig. 2b centre) results in a straight and non-helical structure, which could be explained by aggregation/attachment of primary orthorhombic particles in a less favourable but probable way (Supplementary 

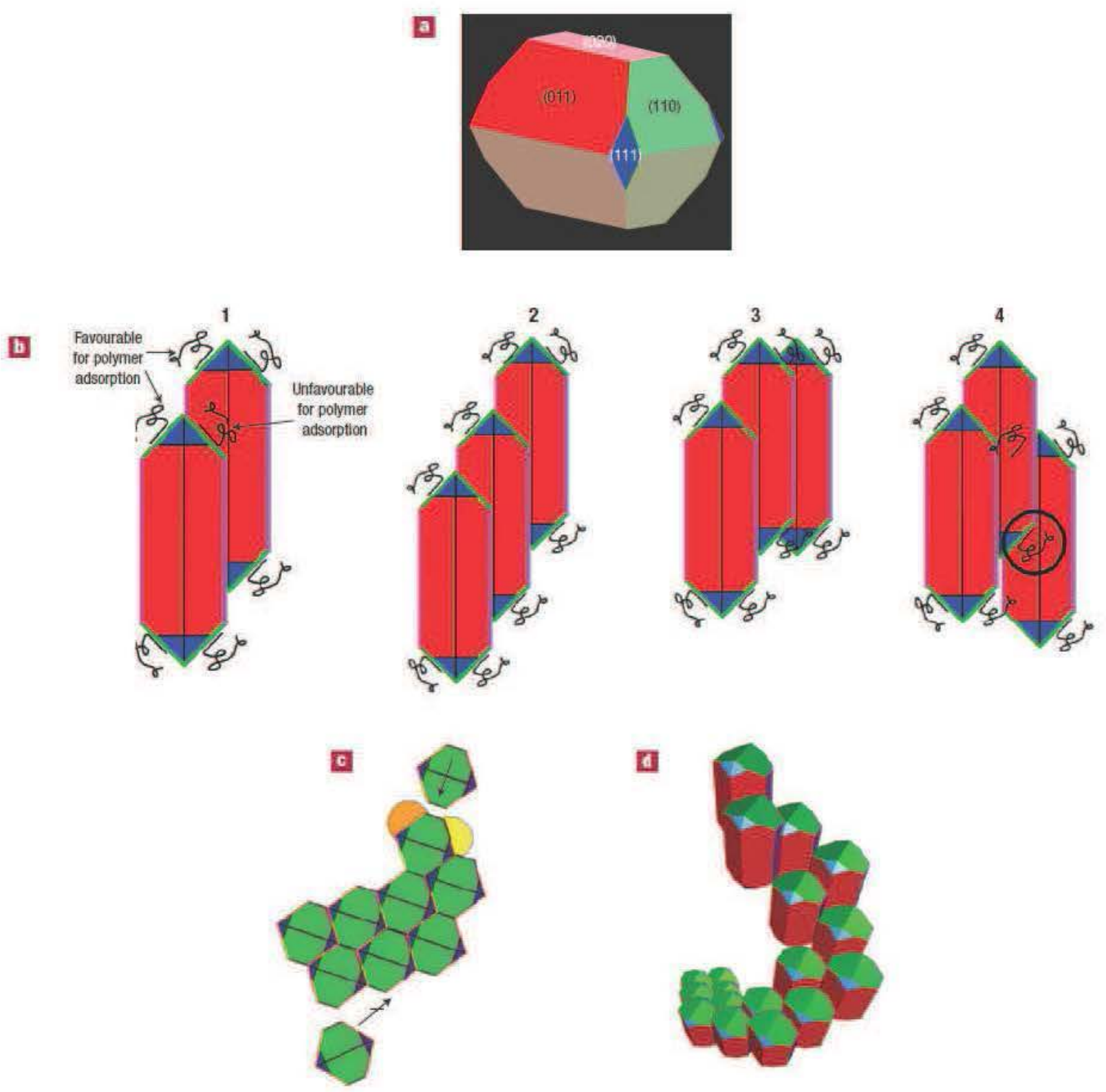

Figure 4 Proposed mechanism leading to spontaneous helix formation. a, Primary nanocrystalline witherite building block in vacuum not representing abserved face areas in solution but just illustrating the orientation of the relevant faces. Colours for the figure: $(110)=$ green, $(111)=$ blue, $(011)=$ red and $(020)=$ pink. b, Interparticle aggregation along the (011) and (020) faces makes the adsorption faces at the tip different, allowing the particles to differentiate between favourable and unfavourable arrangements. The (partially) staggered arrangement is preferred ( 2 and 3 , with 3 being much more improbable) over the probable but energetically excluded arrangernent with unfavourable polymer adsorption sites (circle shown in 4) and predetermines addition of subsequent particles resulting in directed aggregation of subsequent particles either upwards or downwards as defined by the first three aggregated particles. c. Perpendicular view along the helix growth axis: the alternation of particle aggregation by (011) (red lines) and (020) (black lines) faces breaks the linear character and brings in helicity (only one (020) face sterically accessible). The yellow and orange spots indicate different (011) faces for an attaching particle. The orange spot is more favourable for subsequent particle attachment and thus, the helix turn is continued. Green $=(110)$, blue $=(111)$. Overlay of processes $b$ and $c$ leads to the helical superstructure $d$, which even tolerates some mismatched particles.

Information, Fig. $\mathrm{S6}$ ). When the starting $\mathrm{pH}$ was kept at 5,6, the fibres tend to be straighter and the helical structures almost cannot be identified, as shown in Fig. 3a (salt-stick morphology) as a result of an increased axial helix growth.

On the other hand, when the polymer concentration reaches $2 \mathrm{gl}^{-1}$, the previously aligned nanocrystals assembled into short parallel fibre bundles (Fig. 3b). In these aligned structures, the axial growth of the helical superstructures is suppressed resulting in a restricted alignment of the tectons in two dimensions. These results imply that the forces that hold the primary nanocrystals together do not only have components for the mutual vectorial alignment towards fibre bundles (consistent with the previous observation for $\mathrm{BaSO}_{4}$ and $\mathrm{BaCrO}_{4}$ fibre bundles using a simpler $\mathrm{DHBC}$, $\mathrm{Br}^{7.27}$ or low molecular polyelectrolytes ${ }^{27.28}$ ), but also chiral components.

A potential mode for the creation of helicity by the interaction of primary components is suggested (Fig. 4). By wide-angle 
X-ray scattering of the helical superstructures (Supplementary Information, Fig. S3), the witherite structure is unequivocally identified. Calculation of the witherite equilibrium morphology in vacuum using Cerius $^{2}$ suggests the morphology for the primary building nanocrystals depicted in Fig. 4a. The calculated detailed surface patterns of the cleavage planes (Supplementary Information, Fig. S5) show that the positive (110) faces are favourable for the adsorption of the negative phosphonated block copolymer. All other faces are either neutral and/or have an unfavourable tilted positioning of the carbonate anions, which would require a tilted arrangement of the functional polymer groups on adsorption. This would cost conformational entropy, and is thus unfavourable as known from $\mathrm{CaCO}_{3}$ grown on functional monolayers, where the interacting carboxyl groups were always aligned in plane with the carbonate ions of the nucleated faces ${ }^{29}$.

Molecular modelling of the functional polymer block reveals a stiff structure with the functional groups located predominantly on one side, with a distance of the phosphonate moieties fitting to the (110) carbonate packing motif (Supplementary Information, Fig. S1). Selective polymer adsorption to the (110) face (green in Fig. 4) can therefore explain the restricted growth in this direction as well as a selective sterical stabilization of this face. The (110) face is therefore blocked against aggregation, whereas this is not the case for (020) and (011). The (111) faces are too small to become important for any further particle attachment.

The tip-like ending of the crystals plus the polymer rigidity is presumably the mechanistic base for the helical coiling of the inorganic superstructures, as the (110) adsorption sites on the tips become different due to interparticle aggregation (see favourable and unfavourable structures in Fig. 4b). If the steric stress created by the adsorbed stiff polymer is high enough, the system can differentiate between an entrapped 'convex' (unfavourable) and an outside 'concave' (favourable) situation (Fig. $4 \mathrm{~b}$, part 1) for the alignment of the next particle. This is the reason for the preference of staggered arrangements and the subsequent directed aggregation of further nanoparticles along the common axis. In this picture, it is a key for helix formation that the system is orthorhombic. The lateral growth of the superstructure can proceed through oriented attachment of the (011) or (020) surfaces (red and pink in Fig. 4), as known from hydrothermal particle synthesis ${ }^{30}$. Owing to reasons of epitaxy, just (011) - (011) and (020)-(020) face fusion can occur, whereas (011)(020) binding does not take place (Fig. 4c crossed arrow orientation of approaching particle, and Supplementary Information, Fig. S5). Furthermore, the aggregation of the (011) faces is preferred over that of the $(020)$ faces, because each building block contains four (011) faces as compared with two $(020)$ faces.

Any change from $(020)-(020)$ to $(011)-(011)$ aggregation unequivocally leads to a helical bend, as the $(020)$ surfaces of the superstructure aggregating with the next particle are sterically different (Fig. 4c). In addition, also the (011) faces are different for further growth, with the favourable situation indicated by the orange spot in Fig. 4c. It has to be mentioned that the helix turn is already set by the first particles and only propagates afterwards so that a reverse helix turn was never observed. Branching of the helix, which would be possible according to Fig. 4 , is not observed as a result of the fast helix growth, depleting the regions parallel to the helix axis of building units so that primary particle attachment takes place at the helix tips.

The random character of particle side-by-side attachment leads to the non-constant helix pitch observed in all scanning electron microscope (SEM; LEO 1550-Gemini) pictures and the observed equal number of left-handed and right-handed helices. It is however worth mentioning that for primary nanocrystals with a still lower symmetry (that is, tri- and monoclinic minerals), such a process can even lead to the preference of one chiral species.
A pre-requisite for the whole process is that polymer adsorption and particle attachment take place at about the same timescale. At increased starting $\mathrm{pH}$ and the related lower overall charge of the nanocrystals (isoelectric point of $\mathrm{BaCO}_{3}=\mathrm{pH} 10-10.5$ ), the helices become dense and stiff (Fig. 3a), indicating that particle attachment prevails over polymer adsorption. Increasing the polymer concentration and adjusting to starting $\mathrm{pH} 4$ leads to the reverse process, the lateral growth is restricted, and only short parallel arranged fibre bundles are formed (Fig. 3b and Supplementary Information, Fig. S4d).

Although helix formation by self-assembly of macromolecules and organic tectons is $k^{2}$ wn $^{24}$, spontaneous helicity by directed tectonic assembly of inorganic particles and in the absence of packing constraints as reported here is a new mechanism of helix formation. Moreover, on the mesoscale, new modes of spontaneous symmetry breaking, as non-homogeneous polymer adsorption, can obviously be activated, generating chiral contributions in the mutual interaction potentials of the building blocks.

\section{References}

1. Mann, S. The chemistry of form. Angew. Chem. Int, Edn 39, 3392-3406 (2000).

2. Colfen, H. \& Mann, S. Emergence of higher-order organization by mesoscale self-assembly and transformation of hybrid nanostructures. Angew. Chem. Int. Edn 42, 2350-2365 (2003).

3. Estroff, L. A. \& Hamilton, A. D. At the interface of organic and inorganic chemistry: Bioinspired synthe sis of composite materials Chem. Mater. 13, 3227-3235 (2001),

4. Colfen, H. Double-hydrophilic block copolymers: Synthesis and application as novel surfactants and crystal growth modifiers. Macromol. Rapid Commun. 22, 219-252 (2001).

5. Antonietti, M. et al. Inorganic/organic mesostructures with complex architectures: Precipitation of calcium phosphate in the presence of double-hydrophilic block copolymers. Chem. Eur. J. 4, 2493-2500 (1998).

6. Marentette, J. M., Norwig, J., Stockelmann, E, Meyer, W. H. \& Wegner, G. Crystallization of CaCO, in the presence of PEO-block-PMAA copolymers. Adv. Mater. 9, 647-651 (1997).

7. Yu, S. H, Colfen, H. \& Antonietti, M. Control of the morphogenesis of barium chromate by using double-hydrophilic block copolymers (DHBCs) as crystal growth modifiers. Chem. Eur. J. 8, 2937-2945 (2002)

8. Qi, L, M., Colfen. H. \& Antonietti, M. Crystal design of barium sulfate using double-hydrophilic block copolymers. Angew. Chem. Int, Edn 39, 604-607 (2000).

9. Robinson, K. L, Weaver, I. V. M., Armes, S. P., Marti, E. D. \& Meldrum, E. C. Synthesis of controlledstructure sulfate-based copolymers via atom transfer radical polymerisation and their use as crystal habit modifiers for $\mathrm{BaSO}_{4}$ J. Mater. Chem, 12, 890-896 (2002).

10. Xia, Y. et al. One-dimensional nanostructures: synthesis, characterization, and applications. Adv. Mater, 15, 353-389 (2003).

11. Sone, E. D., Zubarev E. R. \& Stupp, S. I. Semiconductor nanohelices templated by supramolecular ribbons. Angew, Chem. Int Edn 41, 1706-1709 (2002).

12. Zhang, H. F, Wang, C. M. \& Wang, L. S. Helical crystalline $\mathrm{SiC} / \mathrm{SiO}_{2}$ core-shell nanowires, Nano Lett. 2, 941-944 (2002).

13. Sato, L. et al. Highly enantioselective synthesis of organic compound using right- and left-handed helical silica. Tetrahedr. Lett. 44, 721-724 (2002).

14. van Bommel, K. J. C., Friggeri. A. \& Shinkai, S. Organic templates for the generation of inorganic materials. Angew. Chem. Int. Edn 42, 980-999 (2003).

15. Sugawara, I, Suwa, Y., Ohkawa, K. \& Yamamoto, H. Chiral biomineralization: Mirror-imaged helical growth of calcite with chiral phosphoserine copolypeptides, Macromol, Rapid Commun, 24, 847-851 (2003).

16. Gower L. A. \& Tirrell, D. A. Calcium carbonate films and helices grown in solutions of poly(aspartate). J. Crystal Growth 191, 153-160 (1998).

17. Garcia-Ruiz, J. M. et al. Self-assembled silica-carbonate structures and detection of ancient microfossils. Science 302, 1194-1197 (2003).

18. Garcia-Ruiz J. M. \& Amoros J. L. Crystal aggregates with induced mor phologies grown by silica gel technique. Bull. Mineral. 104, 107-113 (1981).

19 Imai, H. \& Oaki, Y. Emergence of morphology chirality from twinned crystals. Angew. Chem. Int. Edn 43, 1363-1368 (2004)

20. Terada, T, Yamabi, S. \& Imai, H, Formation process of sheets and helical forms consisting of strontium carbonate fibrous crystals with silicate. J. Crystal Growth 253, 435-444 (2003).

21. Tauer, K, Antonietti, M., Rosengarten, L. \& Maller, H. Initiators based on poly(ethylene glycol) for starting heterophase polymerizations: generation of block copolymers and new particle morphologies. Macromol Chem. Phys, 199, 897-908 (1998).

22. Odian, G. Principles of Polymerization, Third Edition $625-626$ (Wiley, New York, 1991).

23. Sommerdijk, N. A. J. M., Holder, S. J., Hiorns, R. C., Jones, R. G. \& Nolte, R. J. M. Self-assembled structures from an amphiphilic multiblock copolymer containing rigid semiconductor segments. Macromolecules 33, 8289-8294 (2000).

24. Cornelissen, J. J. L. M., Rowan A. E, Nolte R. J. M. \& Sommerdijk, N. A. J. M. Chiral architectures from macromolecular building blocks. Chem. Rev. 101, 4039- 4070 (2001).

25. Weiner, S., Albeck, S. \& Addadi, L. Polysaccharides of intracrystalline glycoproteins modulate calcite crystal growth in vitro. Chem. Eur. J. 2, 278-284 (1996) 
26. Tang, Z. Y., Kotov, N. A. \& Giersig. M. Spontaneous organization of single CdTe nanoparticles into luminescent nanowires. Science 297, 237-240 (2002).

27. Qi, L. M, et al. Formation of $\mathrm{BaSO}_{4}$ fibres with morphological complexity in aqueous polymer solutions, Chem. Eur. J. 7, 3526-3532 (2001).

28. Yu, S. H., Antonietti, M., Colfen, H. \& Hartmann, J. Growth and self-assembly of $\mathrm{BaCrO}_{4}$ and $\mathrm{BaSO}_{4}$ nanofibers toward hierarchical and repetitive superstructures by polymer-controlled mineralization reactions. Nano Lett. 3, 379-382 (2003).

29. Travaille, A. M. et al. Aligned growth of calcite crystals on a self-assembled monolayer. Adv. Mater. $14,492-495$ (2002).

30. Penn, R. L. \& Banfield, J. F. Morphology development and crystal growth in nanocrystalline aggregates under hydrothermal conditions: Insights from titania, Geochim. Cosmochim. Acta 63 , $1549-1557$ (1999).
Acknowledgements

We thank the Max Planck Gesellschaft and the Deutsche Forschungsgemeinschaft for financial support S. H. Yu thanks the special funding support from the Chinese Academy of Sciences the Distinguished Youth and Team Funds from the National Science Foundation of China (No. 20325104, No. 20321101), and NSFC No. 50372065, AvH Foundation, and Max Planck Society). The authors thank the Ivoclar AC (Schaan, Liechtenstein) for the gift of the (2-[4-dihydroxy phosphoryl]-2-oxabutyl) monomer. A. Vorlkel is acknowledged for the ultracentrifuge experiments.

Correspondence and requests for materials should be addressed to S.H. Y, H.C. or M.A.

\section{Competing financial interests}

The authors declare that they have no competing financial interests. 